\title{
Unusual echocardiographic evidence of hypercoagulation in usual left atrial appendage as the first and only sign of COVID-19
}

\author{
M. Świątczak@, R. Nowak, A. Faran, E. Wabich, G. Raczak, M. Klimkiewicz, \\ L. Daniłowicz-Szymanowicz@ \\ Department of Cardiology and Electrotherapy, Medical University of Gdansk, Poland \\ [Received: 14 April 2021; Accepted: 20 May 2021; Early publication date: 25 May 2021]
}

Coronavirus disease 2019 (COVID-19) is a condition caused by a novel virus, severe acute respiratory syndrome coronavirus 2 (SARS-CoV-2). The disease's course ranges from entirely asymptomatic to severely ill patients. Hypercoagulation is often a complication of this disease, worsening the prognosis, which is extremely important in patients at higher risk of thromboembolic events, such as atrial fibrillation (AF), where thrombus formation in the left atrial appendage (LAA) is frequent. LAA could be of various sizes, volumes, and shapes, distinguish several morphologies, from which the WindSock LAA is the most frequent. In contrast, thromboembolic complications occur most frequently in patients with $A F$ and the Cactus LAA. We present a clinical case of a 70-year-old woman with an initial negative real-time polymerase chain reaction (RT-PCR) test for SARS-CoV-2, suspicion of device-related infection after dual pacemaker implantation, $A F$, and LAA without thrombus in the initial transoesophageal echocardiography (TEE). Despite apixaban treatment, spontaneous restoration of sinus rhythm, and WindSock LAA morphology, the sludge in LAA was diagnosed in control TEE. The patient did not present any typical clinical COVID-19 symptoms but re-checked the RT-PCR test for SARS-CoV-2 infection was positive. The described case presents echocardiographic evidence of hypercoagulation as the first and only feature of SARS-COV-2 condition besides the usual morphological presentation of the WindSock LAA. (Folia Morphol 2021; 80, 3: 714-717)

Key words: COVID-19, SARS-CoV-2, coronavirus, atrial fibrillation, left atrial appendage

\section{INTRODUCTION}

Coronavirus disease 2019 (COVID-19) is a condition caused by a novel virus, severe acute respiratory syndrome coronavirus 2 (SARS-CoV-2) [10]. The real-time polymerase chain reaction (RT-PCR) test is the most reliable in diagnosing COVID-19. The disease's course ranges from entirely asymptomatic to severely ill patients. Hypercoagulation is often a complication of this disease, worsening the prognosis [3]. This complication seems to be aggravated in patients at higher risk of thromboembolic events, such as patients with atrial fibrillation (AF), in whom a thrombus formation in the left atrial appendage (LAA) is frequent. Additional factors that increase

Address for correspondence: Ludmiła Daniłowicz-Szymanowicz, MD, PhD, Department of Cardiology and Electrotherapy, Medical University of Gdansk, ul. Dębinki 7, 80-211 Gdańsk, Poland, tel: +58 34939 10, fax: +58 34939 20, e-mail: ludwik@gumed.edu.pl

This article is available in open access under Creative Common Attribution-Non-Commercial-No Derivatives 4.0 International (CC BY-NC-ND 4.0) license, allowing to download articles and share them with others as long as they credit the authors and the publisher, but without permission to change them in any way or use them commercially. 


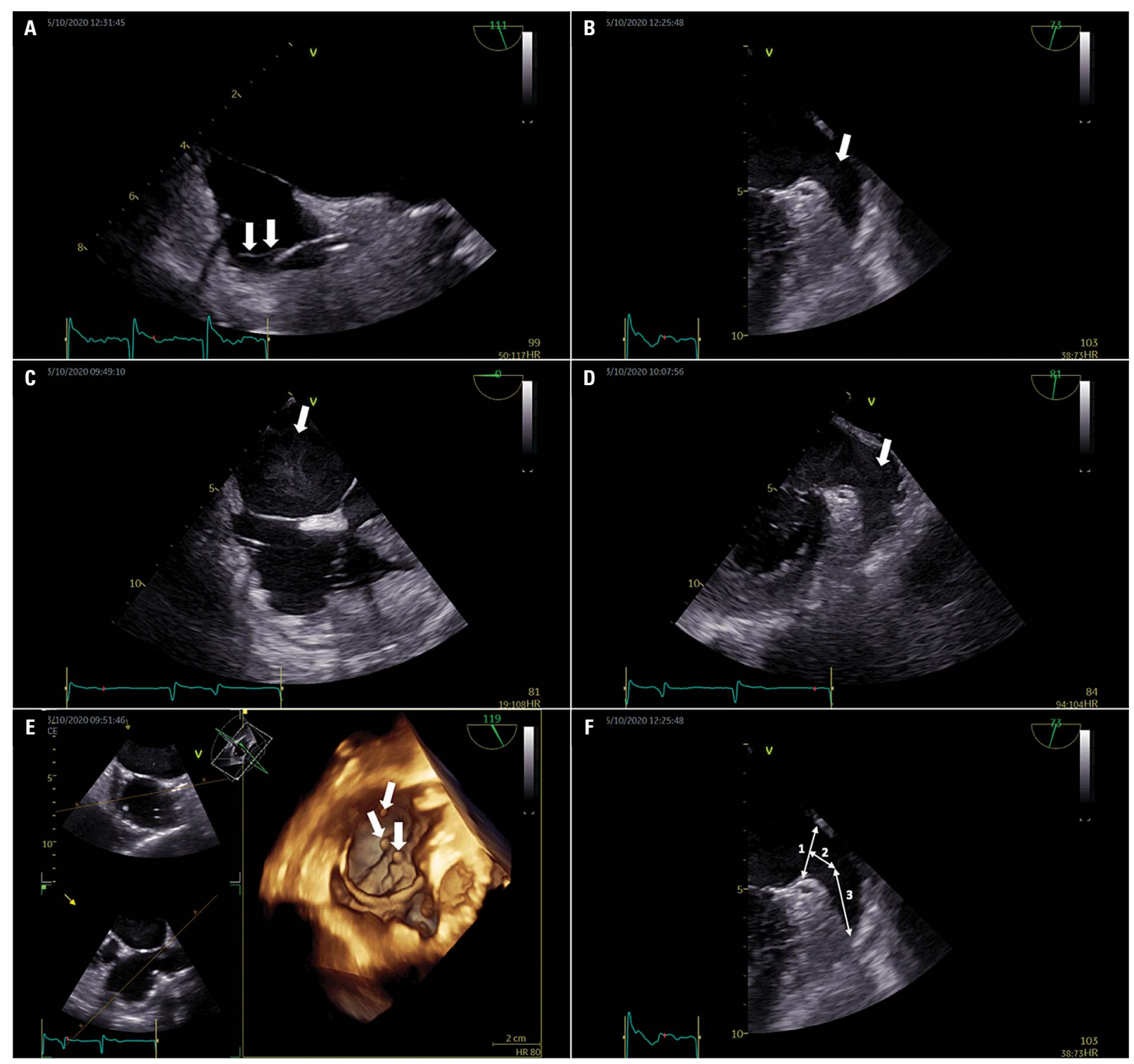

Figure 1. The figures visualise the image in transoesophageal echocardiography performed twice in the described patient. On admission to the Cardiology Department, the patient had linear echoes on the atrial electrode (panel A - arrows) and a clean left atrial appendage (LAA) (panel B - arrow). In a follow-up study after the spontaneous return of sinus rhythm and a week of apixaban (5 mg BID) therapy, the linear echoes on the atrial lead disappeared, while the left atrium filled with highly hyperechogenic blood (panel C — arrow) and LAA with the sludge (panel D - arrow) were visualised. Additionally, the long persistence of the bubbles of midazolam in the right atrium was observed (panel E - arrows). Panel F presents the dimensions of the LAA in the described patient; $1-1.7 \mathrm{~cm}, 2-0.8 \mathrm{~cm}, 3-2.4 \mathrm{~cm}$.

the risk of formation of thrombus in the LAA are the specific anatomy of the LAA and the low left ventricular ejection fraction. We present a clinical case that presents SARS-CoV-2 infection as an additional cause of LAA thrombus formation.

\section{CASE PRESENTATION}

A 70-year-old woman with AF and negative RT-PCR test for SARS-CoV-2 was transferred from the district hospital to the Cardiology Department with suspicion of device-related infection after dual pace- maker implantation, performed 1 week before the admission. Additionally, the urinary tract infection was diagnosed, and empirical antimicrobial therapy was initiated, leading to a quick reduction of inflammatory markers. There were no pathologies connected with ventricle lead; however, in transoesophageal echocardiography (TEE), using two- and three-dimensional techniques, linear echoes associated with the atrial electrode were visualised (Fig. 1A). Due to the short time after the implantation and negative blood culture results, the thrombus was anticipated, 


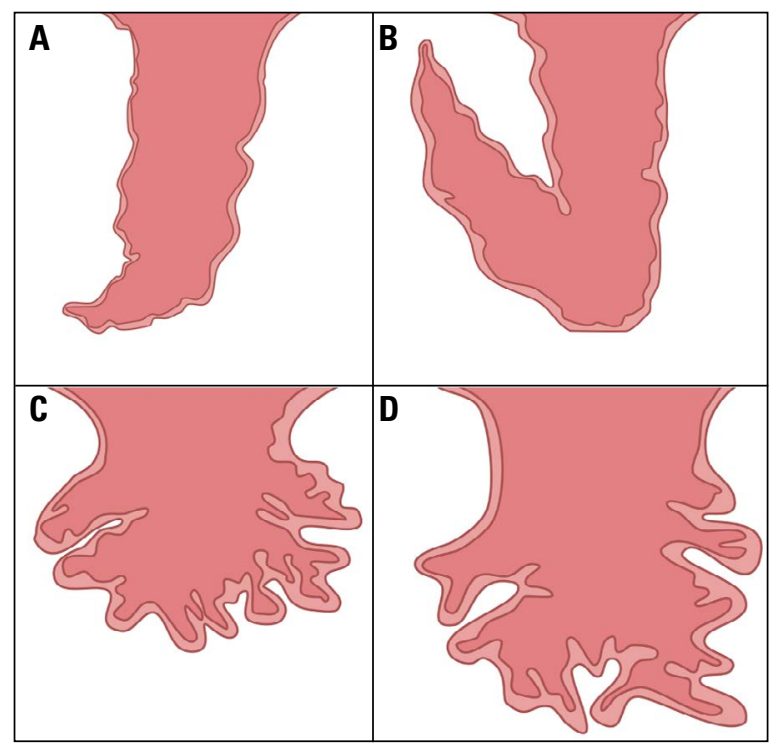

Figure 2. General scheme showing the four most common types of the left atrial appendage (LAA); $\mathbf{A}$. The WindSock LAA; B. The ChickenWing LAA; C. The Cauliflower LAA; D. The Cactus LAA.

not bacterial vegetation. The LAA had typical morphology and sizes, as presented in Figure 1. Despite $A F$, there was no sludge or thrombus in the LAA (Fig. 1B). The spontaneous restoration of sinus rhythm was observed. The treatment with apixaban $(5 \mathrm{mg}$ BID) was initiated, and empirical antibiotic therapy was continued. After 1 week of the treatment, TEE was repeated with no linear echoes within the atrial electrode. Interestingly, despite permanent sinus rhythm and anticoagulation therapy with apixaban, the left atrium was filled with highly hyperechogenic blood (Fig. 1C) and the LAA with a sludge (Fig. 1D). Moreover, the long persistence of the bubbles of midazolam in the right atrium has been observed (Fig. 1E). The patient did not present any typical clinical COVID-19 symptoms [2], but had neutropenia, lymphopenia, low procalcitonin, hypoalbuminaemia, and increased C-reactive protein. We re-checked the RT-PCR test for SARS-CoV-2 infection, and the result was positive.

\section{DISCUSSION}

The LAA lies within the pericardium, in the left atrioventricular sulcus atop the left circumflex artery's proximal part and extends between the anterior and the lateral walls of the LA near the left pulmonary veins [11]. The LAA could be of various sizes, volumes, and shapes and often has several lobes $[5,8,11]$. Veinot et al. [11] found that the most common is the presence of $2(54 \%)$ lobes, the second most frequent are an LAA with $3(23 \%)$ lobes, then with $1(20 \%)$ lobe and the least frequent is the presence of $4(3 \%)$ lobes. The orifice of the LAA leads through the neck to the central appendage cavity, which can have a different shape depending on the type of an LAA. According to Wang et al. [12], the most frequent LAA anatomy is the WindSock LAA (Fig. $2 A$ ), which has no obvious bands, and one dominant lobe of sufficient length is the basic structure. Variations of this type of LAA appear with the different locations and number of secondary lobes descending downward from the dominant lobe. The second most frequent anatomy is the Cauliflower LAA (Fig. 2C), which, like the previous type, does not have obvious bands; an LAA is characterized by a limited overall length and more complex internal features. Its varieties are described by a more irregular shape of the orifice of the LAA, the number of significant lobes, and the lack of the dominant lobe. The ChickenWing LAA (Fig. 2B) is the third most common type; the main feature of that type of LAA is an obvious band in the proximal or middle part of the dominant lobe or a backward fold of the LAA at some distance from the orifice of the LAA. This type may differ in the presence or absence of additional lobes or twigs, with a different measured distance to this bend, and with different orientations of the bends to the main lobe. The last most common type is the Cactus LAA (Fig. 2D); its main feature is the dominant lobe with secondary lobes extending from the dominant lobe to the superior and inferior directions [12].

According to the literature, thromboembolic complications occur most frequently in patients with $\mathrm{AF}$ and the Cactus LAA [4]. Additionally, the small size of LAA, the presence of secondary lobes, the narrow orifice of the LAA, and excessive trabeculations result in low LAA peak flow velocities that could significantly increase the risk of thrombus formation LAA [1]. It should also be emphasized that the large size of the left atrium and the reduced left ventricular ejection fraction are additional risk factors for the development of thromboembolic complications.

In the described case, we presented echocardiographic evidence of hypercoagulation as the first and only feature of SARS-CoV-2 infection in the usual morphological presentation of the WindSock LAA (Fig. 1F). Despite the use of apixaban treatment, the lack of features promoting thrombus formation in the LAA (Fig. 1D), such as excessive trabeculations, a narrow junction of the proximal lobe of the LAA, a narrow junction between the distal lobe of the LAA 
and its proximal lobe, or the presence of additional LAA lobes, there has been a sludge in the LAA. The coagulation abnormalities in COVID-19 are postulated to result from acute inflammation in the organism and increased activity of inflammatory mediators [7]. The urinary tract infection could additionally attenuate the hypercoagulation status in our patient.

\section{CONCLUSIONS}

The connection between hypercoagulation features and COVID-19 in a patient without other typical infection indicators seems to be particularly difficult, as in the presented case. Based on the available literature about COVID-19 management, low-molecular-weight heparin should be considered for thromboembolic complications prophylaxis [6]. In contrast, oral anticoagulant therapy is not recommended due to its limited effectiveness, confirmed in our patient. The LAA in the case presented above did not show any features predisposing to thrombus formation; the LAA was of standard size and not narrow. Besides, a thrombus developed despite the patient's persistent sinus rhythm and anticoagulant treatment, which suggests a hypercoagulability state in the course of COVID-19. The presented case additionally shows that a negative test for SARSCoV-2 infection does not always give a full guarantee that the patient is not infected, and the patient's clinical manifestation should be taken into account in further clinical decisions. It is worth emphasizing that modern echocardiography, including three-dimensional techniques, can be recognised as a part of comprehensive imaging technology that could be helpful in COVID-19 diagnosis [9].

Conflict of interest: None declared

\section{REFERENCES}

1. Al-Saady NM, Obel OA, Camm AJ. Left atrial appendage: structure, function, and role in thromboembolism. Heart. 1999; 82(5): 547-554, doi: 10.1136/hrt.82.5.547, indexed in Pubmed: 10525506.

2. Carfi $A$, Bernabei $R$, Landi $F$, et al. Persistent symptoms in patients after acute COVID-19. JAMA. 2020; 324(6): 603, doi: 10.1001/jama.2020.12603.
3. Connors J, Levy J. Thromboinflammation and the hypercoagulability of COVID-19. J Thromb Haemost. 2020; 18(7): 1559-1561, doi: 10.1111/jth.14849.

4. Di Biase L, Santangeli $P$, Anselmino $M$, et al. Does the left atrial appendage morphology correlate with the risk of stroke in patients with atrial fibrillation? Results from a multicenter study. J Am Coll Cardiol. 2012; 60(6): 531-538, doi: 10.1016/j.jacc.2012.04.032, indexed in Pubmed: 22858289.

5. Kamiński R, Kosiński $A$, Brala $M$, et al. Variability of the left atrial appendage in human hearts. PLoS One. 2015; 10(11): e0141901, doi: 10.1371/journal.pone.0141901, indexed in Pubmed: 26544191.

6. Marietta M, Vandelli P, Mighali P, et al. COVID-19 HD Study Group. Randomised controlled trial comparing efficacy and safety of high versus low Low-Molecular Weight Heparin dosages in hospitalized patients with severe COVID-19 pneumonia and coagulopathy not requiring invasive mechanical ventilation (COVID-19 HD): a structured summary of a study protocol. Trials. 2020; 21(1): 574, doi: 10.1186/s13063-020-04475-z, indexed in Pubmed: 32586394.

7. Panigada $\mathrm{M}$, Bottino N, Tagliabue $\mathrm{P}$, et al. Hypercoagulability of COVID-19 patients in intensive care unit: A report of thromboelastography findings and other parameters of hemostasis. J Thromb Haemost. 2020; 18(7): 1738-1742, doi: 10.1111/jth.14850, indexed in Pubmed: 32302438.

8. Panyawongkhanti M, Fuktongphan P, Chentanez V. Morphometric study of the left atrial appendage related to closure device deployment: a cadaveric study in Thai population. Folia Morphol. 2020; 79(1): 79-85, doi: 10.5603/ FM.a2019.0066, indexed in Pubmed: 31162625.

9. Rozwadowska K, Raczak G, Sikorska K, et al. Influence of hereditary haemochromatosis on left ventricular wall thickness: does iron overload exacerbate cardiac hypertrophy? Folia Morphol. 2019; 78(4): 746-753, doi: 10.5603/ FM.a2019.0025, indexed in Pubmed: 30835340.

10. Sławiński G, Lewicka E. What should a cardiologist know about coronavirus disease 2019? Kardiol Pol. 2020; 78(4): 278-283, doi: 10.33963/KP.15302, indexed in Pubmed: 32336069 .

11. Veinot JP, Harrity PJ, Gentile F, et al. Anatomy of the normal left atrial appendage: a quantitative study of age-related changes in 500 autopsy hearts: implications for echocardiographic examination. Circulation. 1997; 96(9): 3112-3115, doi: 10.1161/01.cir.96.9.3112, indexed in Pubmed: 9386182

12. Wang $Y$, Di Biase $L$, Horton RP, et al. Left atrial appendage studied by computed tomography to help planning for appendage closure device placement. J Cardiovasc Electrophysiol. 2010; 21(9): 973-982, doi: 10.1111/j.1540-8167.2010.01814.x, indexed in Pubmed: 20550614 